\title{
Hubble spies a stellar mystery
}

\section{Exploding star seems unlike any kind known.}

\section{Ron Cowen}

21 April 2015

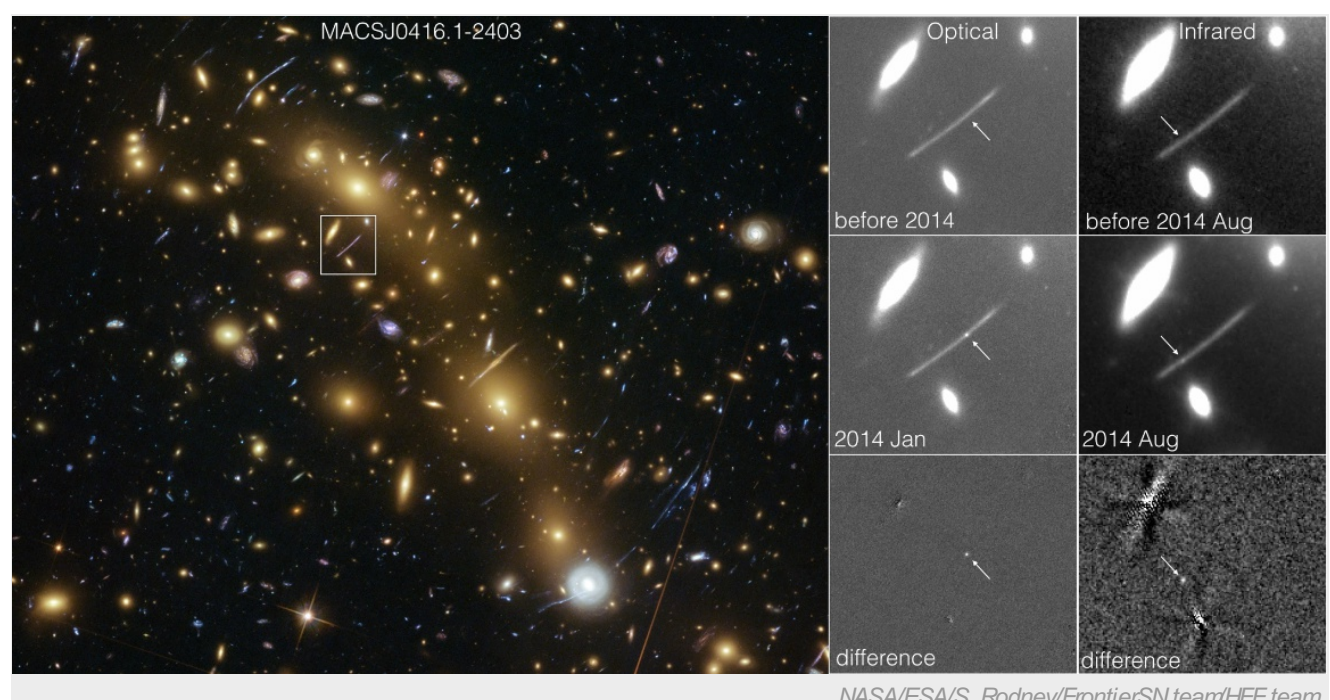

The Hubble Space Telescope captured a stellar outburst in the constellation Eridanus (left) in January and August 2014 (right).

Astronomers have discovered a mysterious stellar outburst that appears to defy classification.

The exploding star, which was seen in the constellation Eridanus, faded over two weeks - much too rapidly to qualify as a supernova. The outburst was also about ten times fainter than most supernovae, explosions that destroy some or all of a star. But it was about 100 times brighter than an ordinary nova, which is a type of surface explosion that leaves a star intact.

"The combination of properties is puzzling," says Mario Livio, an astrophysicist at the Space Telescope Science Institute in Baltimore, Maryland. "I thought about a number of possibilities, but each of them fails" to account for all characteristics of the outburst, he adds.

A team led by Steven Rodney, an astronomer at Johns Hopkins University in Baltimore, observed the puzling event in January and August 2014 with the Hubble Space Telescope. Rodney presented the findings on 21 April at a symposium in Baltimore to celebrate Hubble's 25th anniversary.

The researchers came across the event while participating in a Hubble project that uses galaxy clusters as 'gravitational lenses' to brighten, and collect multiple images of, galaxies that lie beyond each target cluster. This makes it easier to spot faint, ephemeral events such as explosions in distant galaxies. (Rodney's team

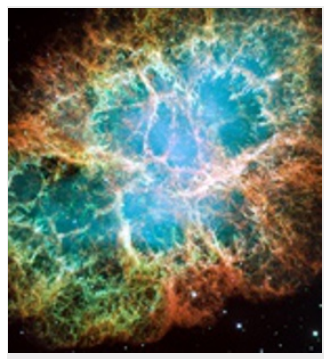

Nature special: Hubble's 25th anniversary last year used the same technique to produce a kaleidoscopic image showing four views of a supernova exploding ${ }^{1}$.)

\section{Ancient light}

The light that Hubble recorded from the newly found outburst left its distant home galaxy 7.8 billion years ago. The scientists are not sure whether the Hubble images collected in January and August of last year each show the same event. A preliminary analysis hints that the observations may depict separate incidents, Rodney says. (In the meantime, the researchers have given the event a nickname: "Spock".)

His team has also not ruled out the possibility that the outburst is a kilonova, an explosion generated when two highly compact stars called neutron stars smash into each other. The brightness and rapidity of the outburst observed by Hubble would match that of a kilonova. But kilonova observations are extremely rare, says Rodney. "We'd be very lucky to see one such event," he says. Seeing two in a single year would be hard to explain, he adds. 
Livio cites another potential problem with this explanation. Kilonovae are typically accompanied by a burst of $\mathrm{Y}$-rays or $\mathrm{X}$-rays, but neither NASA's Swift satellite nor the space agency's Chandra X-ray Observatory observed any high-energy radiation during the event, he says.

"This is a peculiar [outburst], that though the analysis is preliminary, challenges our models," says Rodney. If his team's find is an example of a new class of objects that is common in the Universe, then future wide-field surveys conducted with the Large Synoptic Survey Telescope and the proposed Wide Field Infrared Space Telescope might be able to find many more events like this one, Rodney says.

Nature | doi:10.1038/nature.2015.17371

\section{References}

1. Kelly, P. L. et al. Science 347, 1123-1126 (2015). 